Horizons philosophiques

\title{
Du recours heideggerien à la thèse ontologique de Parménide : sur la différence ontologique comme le fait originaire
}

\section{Danic Parenteau}

Volume 14, numéro 2, printemps 2004

Rencontres avec Heidegger

URI : https://id.erudit.org/iderudit/801262ar

DOI : https://doi.org/10.7202/801262ar

Aller au sommaire du numéro

Éditeur(s)

Collège Édouard-Montpetit

ISSN

1181-9227 (imprimé)

1920-2954 (numérique)

Découvrir la revue

Citer cet article

Parenteau, D. (2004). Du recours heideggerien à la thèse ontologique de Parménide : sur la différence ontologique comme le fait originaire. Horizons philosophiques, 14(2), 27-36. https://doi.org/10.7202/801262ar d'utilisation que vous pouvez consulter en ligne. 


\section{DU RECOURS HEIDEGGERIEN À LA THĖSE ONTOLOGIQUE DE PARMÉNIDE : SUR LA DIFFÉRENCE ONTOLOGIQUE COMME LE FAIT ORIGINAIRE}

La différence ontologique, la différence qui sépare être et étant, est l'un des rares concepts fondamentaux de Heidegger à traverser l'ensemble de son œuvre, tant sa première pensée qui est essentiellement contenue dans Être et Temps, que sa seconde pensée qui procède du Tournant (die Kehre). Cette pérennité peut s'expliquer par l'importance de cette différence pour la question de l'être (die Seinsfrage), question directrice de la pensée heideggerienne. Pour Heidegger, articuler la question de l'être, c'est-à-dire s'enquérir de l'être, consiste à poser son regard sur la différence essentielle qui le sépare de l'étant, soit la différence ontologique. En effet, seule une ouverture à cette différence rend possible une saisie de l'être tel qu'il est véritablement, car «nous ne pensons l'être tel qu'il est que si nous le pensons dans la différence qui le distingue de l'étant et si nous pensons l'étant dans la différence qui le distingue de l'être ${ }^{1 ",}$, écrit Heidegger dans «Identité et différence». L'être ne peut être pensé que dans son rapport - qui est celui d'une différence - avec l'étant, car "être veut dire, toujours et partout : être de l'étant» et "(p)artout et toujours, l'étant veut dire : étant de l'être'"». Ainsi, penser l'être n'est possible qu'à partir de la différence ontologique et en elle.

Curieusement, malgré l'importance de la différence ontologique pour la question de l'être, celle-ci ne fera, de la part de Heidegger, l'objet d'aucun effort de justification quant à son existence. Dans son œuvre pourtant volumineuse, Heidegger s'enquiert rarement, pour ne pas dire presque jamais, de façon directe et explicite de la différence ontologique. Pour importante qu'elle soit, la différence ontologique se fait pourtant très discrète dans la pensée de l'être; elle s'impose d'elle-même, comme allant de soi. En effet, dans les premières lignes de l'ouvrage fondateur de sa pensée, Heidegger introduit cette différence au moyen d'un rappel de l'évidence sur laquelle la pensée ne saurait se méprendre, à savoir que "l'être de l'étant n'"est" pas lui- 
même un étant ${ }^{3}$ ». La différence ontologique est ainsi d'emblée présentée par Heidegger comme un fait que l'on ne saurait réfuter et qui se passe donc de justification quant à son existence. D'ailleurs, semble-t-il considérer, quel intérêt y aurait-il à justifier l'existence de ce qui a déjà été mis en lumière et exploré par les premiers penseurs de l'Occident? On se rappelle que Heidegger considère que les penseurs présocratiques, en particulier Anaximandre, Héraclite ou Parménide, étaient déjà ouverts à la différence qui sépare l'être de l'étant, et que ce n'est que lorsque la pensée devint métaphysique, avec Platon et Aristote, que cette différence fut oubliée : c'est ce que désigne l'idée de l'«oubliance de l'être ${ }^{4}$ ». Aussi, dans ses ouvrages subséquents, Heidegger s'estimera-t-il dispensé de revenir sur cette question et de s'enquérir de l'existence de cette différence. Par suite, celle-ci reste inexplorée dans l'ensemble de sa pensée.

Le présent article se veut une analyse de la place et du rôle de la différence ontologique au sein de l'ensemble de la pensée de Heidegger, tant sa première pensée que sa pensée qui procède du Tournant. Notre travail vise une compréhension de la manière dont Heidegger conçoit la différence ontologique et de l'importance qu'il lui accorde. II s'agira pour nous de montrer pourquoi Heidegger considère que la différence ontologique peut se passer d'une quelconque interrogation quant à son existence, pouvant ainsi être présentée comme relevant de l'évidence. Notre analyse portera premièrement sur la place de la différence ontologique au sein des deux pensées de Heidegger. Deuxièmement, nous analyserons la manière dont Heidegger cherche à faire valoir la différence ontologique comme le fait originaire, c'est-à-dire ce à quoi rien ne saurait échapper et ce sur quoi tout repose. Pour Heidegger, au "fondement" de tout ce qui "est» se trouve la différence ontologique. Troisièmement, nous formulerons une critique de cette idée de la différence ontologique comme le fait originaire.

\section{La différence ontologique au fondement de la pensée de Heidegger}

La différence ontologique tient un rôle fondamental dans l'ensemble de la pensée de Heidegger, dans la mesure où toute sa pensée, aussi bien sa première que sa seconde, repose sur cette différence. D'une part, dans sa première pensée, qui est principalement contenue dans l'œuvre de 1927, Heidegger déploie la question de l'être au moyen d'une analyse de cet étant qui jouit du privilège incomparable 
"d'avoir en son être un rapport d'être5" à l'être, en ce que "(l)e privilège ontique du Dasein consiste en ce qu'il est ontologique ${ }^{6}$ ". II s'agit par cette démarche de "rendre transparent 7 " dans son être le Dasein, dans la mesure où celui-ci est toujours et déjà lié à l'être. Cette démarche de pensée pose ainsi en son fondement l'existence d'une différence entre l'étant, auquel appartient le Dasein, et l'être, soit ce à quoi est lié le Dasein et ce par quoi il se distingue des autres étants. Pareille distinction entre un niveau "ontique" et un niveau "ontologique», distinction qui est à la base du projet contenu dans Etre et Temps, se fonde sur la différence ontologique. La première pensée de Heidegger repose ainsi sur cette différence fondamentale qui sépare être et étant.

D'autre part, la seconde pensée de Heidegger repose, elle aussi, en son fondement sur la différence ontologique. La pensée du Tournant résulte d'un rejet de la conception traditionnelle du rapport entre être et pensée, conception à laquelle souscrit non seulement la métaphysique, cette forme de pensée que Heidegger tente de dépasser, mais également, la pensée philosophique telle qu'elle se déploie dans Être et Temps ${ }^{8}$, ainsi qu'il le reconnaît lui-même. Dans son œuvre de 1927, la question de l'être se déploie comme un effort afin que la pensée puisse cheminer en direction de l'être : la pensée est alors une pensée qui va vers l'être, une pensée sur l'être. Le Tournant dans la pensée heideggerienne se veut un renversement de cette conception du rapport entre pensée et être, conception qui voit dans l'être un "objet" pour la pensée. Le penseur du Tournant estime que c'est bien plutôt l'être qui est à l'origine de la pensée, car celle-ci est une manifestation de l'être. Dans la Lettre sur l'humanisme, Heidegger décrit ainsi sa seconde pensée comme une pensée de l'être, c'est-à-dire une pensée qui émane de l'être, suivant le sens subjectif du lien génitif qui lie l'être à la pensée ${ }^{9}$. La pensée est un "événement ${ }^{10}$ " de l'être lui-même, écrit Heidegger dans "Qu'est-ce que la métaphysique?». Cette conception de la pensée comme manifestation de l'être traduit ainsi un renversement profond de la conception traditionnelle du rapport entre être et pensée, conception suivant laquelle la pensée n'étant saisie que dans son rapport avec le sujet pensant, ne désigne qu'une capacité propre à un étant particulier, à savoir l'être humain. Traditionnellement, la pensée désigne précisément ce qui distingue l'être humain parmi les étants : l'être humain est celui qui pense, il est celui qui est doté de la capacité de penser. La pensée est ainsi liée à l'étant : elle se déploie en direction de l'être, 
comme si l'être était quelque chose d'extérieur et d'étranger à elle. Reconnaître la pensée comme manifestation de l'être exige donc un renversement fondamental dans la conception du rapport entre être et pensée, renversement qui n'est lui-même possible que sur la base d'une ouverture à la différence essentielle qui sépare l'être de l'étant, soit d'une ouverture à ce qui "est» essentiellement différent de l'étant. Ainsi, la pensée du Tournant repose, elle aussi, sur la différence fondamentale qui sépare être et étant.

L'ensemble de la pensée de Heidegger repose ainsi sur la différence ontologique. En articulant la question de l'être, aussi bien dans la perspective de la pensée qui est contenue dans Être et Temps, que dans celle à partir de laquelle se déploie la pensée du Tournant, Heidegger s'ouvre à cette différence et fait reposer son entreprise de pensée sur elle.

\section{La différence ontologique comme le fait originaire : Heidegger et la thèse ontologique de Parménide}

Que la différence ontologique puisse apparaître comme relevant de l'évidence, se passant ainsi de justification quant à son existence, et que l'ensemble de la pensée heideggerienne puisse être fondé sur cette différence, cela découle du fait que pour Heidegger, la différence ontologique représente le fait originaire. Dans la pensée de l'être, la différence ontologique est conçue d'une part, comme quelque chose d'indéniable et de manifeste - il y a différence ontologique : cela ne saurait être réfuté ou même passer inaperçu - et d'autre part, comme ce à quoi rien ne saurait échapper, tout ce qui "est" prenant place au sein de cette différence. Dans un langage non heideggerien, nous pourrions dire que pour Heidegger, la «totalité du réel» repose au sein de la différence ontologique, ou bien que la différence ontologique est "au fondement» de tout ce qui est ${ }^{11}$.

Bien que Heidegger ne fournisse dans l'ensemble de son œuvre aucune justification quant à l'existence de la différence ontologique et qu'il ne s'enquière pas directement et explicitement de celle-ci, nous pouvons tout de même déceler dans sa pensée un effort discret, mais non moins manifeste, pour faire valoir la différence ontologique comme le fait originaire. En effet, nous croyons que Heidegger opère d'une manière implicite dans son œuvre un rapprochement entre l'être tel qu'il est conçu chez Parménide - dans le sixième fragment de son

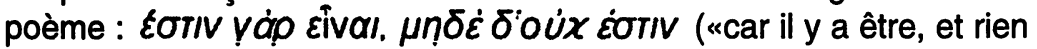
il n'y a pas") $)^{12}$ - et le fait que l'être ne puisse être pensé que par et 
dans la différence ontologique. Par ce rapprochement, Heidegger souhaite que la différence ontologique puisse alors apparaître, à la manière de l'être parménidien, comme ce qui est à la fois évident et indéniable, c'est-à-dire comme ce qui va de soi, se passant donc de justification quant à son existence, et comme ce à quoi rien ne saurait échapper; la différence ontologique pourrait alors apparaître comme le fait originaire.

En raison de l'importance qui revient à Parménide eu égard à la question de l'être, ce penseur occupe une place prépondérante et incomparable dans la pensée de Heidegger. Celui-ci estime que Parménide serait le premier penseur de l'être, le premier à poser la question de l'être et à prendre conscience de ce que Heidegger nomme "le mystère originel pour toute pensée ${ }^{13}$ ". On le sait, pour Heidegger, la suite de l'histoire de la pensée occidentale — pensée qui est devenue avec Platon et Aristote "métaphysique» - ne sera qu'un long déclin, celui de l'oubli de plus en plus grandissant de l'être ${ }^{14}$.

Dans le sixième fragment de son poème, Parménide met pour la première fois en paroles l'émerveillement que suscite la vue de ce fait mystérieux et extraordinaire - de ce fait originaire -, qui est

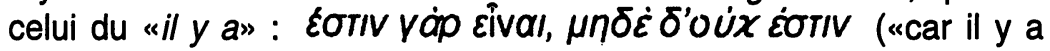
être, et rien il n'y a pas") $)^{15}$. Cette parole renferme deux propositions. D'une part, dans ce poème, Parménide reconnaît à l'être le caractère de l'évidence, à savoir que l'être est quelque chose d'évident et d'indéniable qui ne saurait échapper à la vue : ÉTIV yóo civaıı, car il y a être. D'autre part, plaçant l'être dans un rapport de négation radicale avec le «rien» ou le «non-être» - l'être est tout ce qui n'est pas

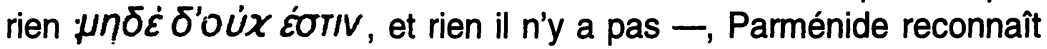
ainsi en plus à l'être de représenter tout ce qui est : rien ne saurait échapper à l'être, à l'exception du rien, qui précisément n'est pas. Tout ce qui est prend place au sein de l'être, fait partie de l'être. Aussi pour Parménide, l'être désigne bien un fait évident et indéniable auquel rien ne saurait échapper : il est le fait originaire.

Heidegger souscrit à cette conception parménidienne de l'être ${ }^{16}$. C'est bien en ce sens, par exemple, qu'il parle de l'être dans Être et Temps, lorsqu'il dit de celui-ci qu'il est : es gibt Sein ${ }^{17}$. Dans son Introduction à la métaphysique, Heidegger reconnaît par ailleurs le rapport de négation que l'être entretient avec le rien ou le non-être : «(q)ue l'être soit différent du non-être, c'est ce dont, en ce cas, nous 
ne doutons pas ${ }^{18}$ ». Bien qu'il souscrive à cette conception parménidienne de l'être, Heidegger va pourtant la développer en lui ajoutant l'exigence de l'horizon de la différence ontologique comme voie d'accès.

En effet, comme nous l'avons souligné déjà, Heidegger considère que l'être ne peut être appréhendé que dans l'horizon de la différence ontologique. Penser l'être n'est possible qu'à partir de cette différence et en elle : "nous ne pensons l'être tel qu'il est que si nous le pensons dans la différence qui le distingue de l'étant 19 ", car l'" être veut dire, toujours et partout : être de l'étant20 ". Penser l'être, c'est donc toujours et déjà avoir devant soi la différence ontologique, c'est toujours et déjà être ouvert à cette différence qui sépare être et étant.

Heidegger soutient donc d'une part, souscrivant à la conception parménidienne de l'être, que l'être constitue à la fois un fait évident et indéniable auquel rien ne saurait échapper - il est le fait originaire et, d'autre part, que ce dernier n'est uniquement accessible que par et dans la différence ontologique. Suivant le raisonnement heideggerien, il s'ensuit que si l'être représente un fait évident et indéniable auquel rien ne saurait échapper, et s'il n'est uniquement accessible que par et dans la différence ontologique, c'est donc que la différence ontologique est elle-même un fait évident et indéniable auquel rien ne saurait échapper. L'être et l'étant, dans leur différence essentielle, représentent ainsi le fait originaire : voilà le raisonnement qui soustend le rapprochement implicite et discret qu'opère Heidegger entre l'être tel qu'il est conçu chez Parménide et l'exigence de la différence ontologique comme seul horizon possible au sein duquel l'être peut apparaître. Par ce rapprochement, Heidegger espère d'une part que soit attribué à la différence ontologique le caractère d'évidence et d'indéniabilité qui est reconnu à l'être dans la thèse ontologique parménidienne. Heidegger considère implicitement qu'à la manière de l'être parménidien, la différence ontologique pourrait se passer d'une quelconque justification quant à son existence : il ne serait pas plus approprié d'exiger une justification quant à l'existence de la différence ontologique, que d'exiger une telle justification pour l'être : car il y a être. L'on peut donc dire que pour Heidegger, «il y a différence ontologique». Par ce rapprochement, Heidegger cherche d'autre part à ce que la différence ontologique soit reconnue comme ce à quoi rien ne saurait échapper. La différence ontologique ne représente pas un simple élément au sein de tout ce qui est, puisqu'elle désigne bien 
elle-même ce à quoi rien ne saurait échapper : tout ce qui est prend place au sein de la différence ontologique. Pour Heidegger, on peut donc dire qu' 'il y a différence ontologique, et non-différence ontologique il n'y a pas". Suivant ce rapprochement, la différence ontologique désignerait donc à la fois ce qui se passe de justification quant à son existence, dans la mesure où elle constitue un fait évident et indéniable, mais aussi ce à quoi rien ne saurait échapper, c'est-àdire ce au sein de quoi tout ce qui est prend place. La différence ontologique constitue bien pour Heidegger le fait originaire ou, comme il qualifie lui-même cette différence dans son Introduction à la métaphysique, «la scission originaire $21 »$.

\section{Une critique de la différence ontologique comme le fait originaire : sur l'interprétation heideggerienne de la thèse ontologique de Parménide}

Or, pareil rapprochement entre la conception parménidienne de l'être et l'exigence de la différence ontologique comme seul horizon possible au sein duquel l'être puisse apparaître est inacceptable, car il repose sur une interprétation irrecevable de ce fragment du Poème de Parménide. Ce qui se dégage du poème parménidien est un émerveillement à la vue du fait qu'il y a quelque chose. Ce poème exprime la prise de conscience non pas du fait qu'il y ait ceci ou cela, mais bien du fait qu'il y a tout court. Le fait qu'il y a quelque chose, Parménide le nomme Eivaı (être). Pour Parménide, l'être ne désigne pas ce qu'il y a, mais bien seulement le fait extraordinaire qu'«il y a". Or, s'émerveiller face à la vue du «il y a", cela n'est pas reconnaître que dans le «il y a" se trouvent deux choses, l'une pouvant être désignée comme "être», l'autre comme "étant», deux choses qui seraient séparées par une différence essentielle. Que l'on puisse reconnaître dans le «il y a» un être et un étant - que l'on puisse y reconnaître la différence ontologique - , cela ne saurait relever du fait qu'«il y a", c'est-à-dire du «il y a" lui-même, mais bien de la manière dont il est possible de saisir et de donner sens à ce qu'il y a dans le «il y a» ${ }^{22}$. Heidegger parle dans Être et Temps de «la première découverte de l'être de l'étant chez Parménide ${ }^{23}$ ", comme si dans la thèse ontologique parménidienne il était dit qu'«il y a être et étant». Mais ce que découvre et affirme Parménide, c'est non pas le fait de l'existence de l'être de l'étant ni encore moins d'une différence essentielle les séparant tous deux, mais bien le fait qu'il y a tout court - ce que Parménide nomme Eivaa (être) -, dans la mesure où l'cêtre» par- 
ménidien ne désigne pas, comme le souligne Marcel Conche dans son étude sur Parménide "ce qu'il y a, mais le il y a lui-même24».

II appert donc que dans sa lecture du poème de Parménide, Heidegger néglige cette distinction pourtant essentielle entre le «il y a» et "ce qu'il y a". En effet, pour Heidegger, les deux termes qui composent le poème de Parménide, soit غ́oTIV et EÑ représentent qu'une seule et même chose. Pour Heidegger, le fait qu'il y a quelque chose et ce qu'il $y$ a ne sont en définitive que le "Même ${ }^{25}$ ". C'est bien en ce sens, que lors du séminaire de Zähringen, Heidegger pourra affirmer au sujet de la thèse ontologique de Parménide qu'elle constitue une "tautologie manifeste26". Ne reconnaissant pas chez Parménide la différence qui distingue ÉסTIV et $\varepsilon \tilde{N} \alpha \mathbf{I}$, Heidegger identifie totalement la différence ontologique non pas à "ce qu'il y a» dans le «il y a", mais bien au «il y a" lui-même. C'est pour cette raison que chez Heidegger, la différence ontologique est conçue comme le fait originaire : «il y a différence ontologique, et non-différence ontologique il n'y a pas".

Pour conclure, ne pouvant trouver appui sur la thèse ontologique de Parménide afin de faire valoir la différence ontologique comme le fait originaire - l'interprétation de la thèse sur laquelle il fonde son rapprochement étant irrecevable - , Heidegger se voit donc incapable de pouvoir justifier l'existence de ce sur quoi il fait pourtant reposer l'ensemble de sa pensée - tant celle qui est contenue dans Être et Temps que celle qui procède du Tournant -, à savoir la différence ontologique. Conséquemment, la différence ontologique chez Heidegger se trouve privée de toute justification quant à son statut fondamental. Heidegger est incapable de faire valoir la différence ontologique comme le fait originaire, c'est-à-dire comme ce qui se présente comme relevant de l'évidence et de l'indéniabilité et comme ce à quoi rien ne saurait échapper. Compte tenu de l'importance de la différence ontologique pour l'articulation de la question de l'être, que cette différence ne puisse apparaître comme le fait originaire ne peut donc être sans conséquences sur la question directrice de cette pensée. En effet, que devient la question de l'être si celle-ci n'est plus liée à un questionnement portant sur le fait originaire?

Danic Parenteau

Université de Paris 1

(Panthéon-Sorbonne) 
Du recours heideggerien à la thèse ontologique de Parménide : sur la différence ontologique comme le fait originaire

1. "Identité et différence», trad. André Préau, in Questions I et ", Paris, Gallimard (coll. Tel), 1968, p. 296, nous soulignons.

2. Idem.

3. Être et Temps, trad. E. Martineau, Paris, Authentica (édition hors commerce), 1985, p. 29 (6). Les chiffres entre crochets indiquent la page de l'édition allemande du texte (Sein und Zeit, Tübingen, Max Niemeyer Verlag, 1979). II faudra attendre la publication en 1929, de "L'être-essentiel d'un fondement ou "raison" pour que cette différence soit explicitement qualifiée par Heidegger de "différence ontologique" (cf. "L'être-essentiel d'un fondement ou "raison"» trad. H. Corbin, in Questions I et II, Paris, Gallimard (coll. Tel), 1968, p. 100).

4. Introduction à la métaphysique, trad. G. Kahn, Paris, Gallimard (coll. Tel), 1967, p. 31.

5. Être et Temps, p. 32 (12).

6. Être et Temps, p. 32 (12), l'auteur souligne.

7. Être et Temps, p. 30 (7).

8. "Séminaire du Thor"(mardi 2 septembre 1969), trad. J. Beaufret, in Questions IV, Paris, Gallimard (coll. Classiques de la philosophie), 1976, p. 268. Voir aussi la Lettre à Jean Wahl de 1937 cité in Pierre Bourdieu, L'ontologie politique de Martin Heidegger, Paris, Éd. de Minuit, 1988, p. 114.

9. Lettre sur l'humanisme, trad. R. Munier, éd. bilingue, Paris, Aubier, 1964, p. 35. Sa première pensée est ainsi également décrite comme une pensée de l'être, mais suivant le sens objectif du rapport génitif entre être et pensée : la première pensée heideggerienne de l'être est la pensée sur l'être, celle qui a l'être comme objet.

10. "Qu'est-ce que la métaphysique?",trad. K. Axelos et J. Beaufret, in Questions I et ", Paris, Gallimard (coll. Tel), 1968, p. 79.

11. Ces deux formules ne sont toutefois pas parfaitement fidèles à la pensée de Heidegger. On sait que Heidegger refuse la conception traditionnelle de la réalité ou du monde, à laquelle il oppose l'idée de "mondanéité" (Weltlichkeit) et d'cêtreau-monde" (In-der-Welt-sein) (cf. Être et Temps, § 14, p. 68 sq. [63 sq.]), et qu'il refuse également de concevoir l'être - et par extension la différence ontologique - comme le ufondement» de ce qui est, soit comme l'étant, car dans cette conception métaphysique, l'être serait conçu non pas en tant que ce qui est essentiellement différent de l'étant, mais bien en tant que simple étant, soit en tant que cet étant qui se trouve au fond de l'étant (cf. Introduction à la métaphysique, p. 15).

12. Nous suivons la traduction donnée par Marcel Conche dans Parménide, Le Poème : Fragments, Paris, P.U.F., 1999, p. 100.

13. Lettre sur l'humanisme, p. 87. Heidegger écrit bien "das anfängliches Geheimnis».

14. Cf. entre autres, Introduction à la métaphysique, p. 203 et Lettre sur l'humanisme, p. 35.

15. Littéralement, ce poème dit "est car être». ÉंTIV signifie "est", soit le verbe être à la troisième personne du singulier de l'indicatif présent. En français, on traduit généralement par le gallicisme «il y a ...", ou plus rarement par l'expression «il est...". En allemand, on dira plutôt "es gibt ...", soit "cela donne ...", ou bien șimplement «ist...", soit "est...", et en anglais, "there is ...", soit " il est... ". ENal signifie "être", soit la forme substantivée du verbe être.

16. George Vick a bien démontré comment Heidegger est parvenu à réhabiliter la 


\section{Danic Parenteau}

notion d'être chez Parménide («Heidegger's Linguistic Rehabilitation of Parmenides' "Being"”, in Michael Murray (dir.), Heidegger and Modern Philosophy. Critical Essays, Londres, Yale University Press, 1978, p. 203-221).

17. Être et Temps, p. 159 (212).

18. Introduction à la métaphysique, p. 86.

19. «Identité et différence», p. 296, nous soulignons.

20. Idem.

21. Introduction à la métaphysique, p. 207, l'auteur souligne.

22. Nous reprenons ici une distinction qu'établit Marcel Conche dans son analyse du poème de Parménide.

23. Être et Temps, p. 159 (212), l'auteur souligne.

24. Parménide, Le Poème : Fragments, p. 103. Par ailleurs, sans vouloir poursuivre plus loin cette analyse du poème de Parménide, il nous semble que s'il fallait reconnaître dans la parole de ce présocratique une quelconque différence, ce ne saurait être celle que relève Heidegger, différence entre "être" et "étant", mais bien plutôt celle entre "être" et «rien», soit entre "il y a» et «il n'y a pas». Parménide dit bien "car il y a être, et rien il n'y a pas" (ibid., p. 100). Le mystère de l'être parménidien consiste bien à s'émerveiller face à la vue du fait qu'il y a quelque chose plutôt que rien.

25. "Séminaire de Zähringen" (samedi 8 septembre 1973), trad. J. Beaufret, in Questions IV, op. cit., p. 336. Heidegger dit bien «das Selben (Gesamtausgabe. I. Abteilung : veröffentliche Schriften 1910-1976, Seminare, Francfort-sur-Main, Klostermann, 1986, vol. 15, p. 397).

26. Heidegger dit bien «eine offenbare Tautologie» (idem.). 